\title{
V type high birefringent PCF fiber for hydrostatic pressure sensing
}

\author{
J. Wojcik, ${ }^{*}$ P Mergo, M. Makara, K. Poturaj, K. Skorupski, and J. Klimek \\ Departament of Optical Fibers Technology, Maria Curie Sklodowska University, \\ Maria Curie Sklodowska Sq3, 20-031 Lublin
}

Received March 22, 2010; accepted March 25, 2010; published March 31, 2010

\begin{abstract}
In the paper a new kind of optical fibres design for application in phase pressure sensors is presented. Fibres were modeled, fabrication technology was developed and the properties of fabricated fibres were measured. The results do not quite agree with those of modeling carried out by using commercially available software.
\end{abstract}

The polarimetric response to temperature in classical highly birefringent (HB) fibres is associated mostly with thermal stress induced by different expansion coefficients in the fiber core and the cladding. Hence, conventional HB fibres are characterized by relatively high values of the polarimetric sensitivity to temperature. In classical side-hole HB optical fibers two empty holes are placed on both sides of the core [1]. Therefore this kind of optical fiber has enhanced sensitivity to pressure up to $100 \mathrm{rad} /(\mathrm{MPa} \cdot \mathrm{m})$ and sensitivity to temperature as low as $0,25 \mathrm{rad} /(\mathrm{K} \cdot \mathrm{m})[1,2]$.

Theoretical analysis and the results of measurements published recently have shown that polarimetric sensitivity to temperature in highly birefringent photonic crystal fibres (PCF) can be even two orders of magnitude lower than that in traditional fibers $[3,4]$.

In this paper we proposed a new type of highly birefringent photonic crystal fiber with filling factor asymmetry. Additionally, by proper selection of fiber geometry enhanced sensitivity to pressure was obtained.

The borderline between large and small holes is of the $\mathrm{V}$-shape, hence the fiber name.

Calculations were made for the same two outside diameters $\Phi$ PCF's with different smaller holes diameters $\mathrm{d}$, larger holes $\mathrm{D}$ and different lattice constants $\Lambda$ (Tab. 1).

\begin{tabular}{|c|c|c|c|c|}
\hline $\begin{array}{c}\text { Geometrical } \\
\text { parameter }\end{array}$ & $\Phi[\mu \mathrm{m}]$ & $\Lambda[\mu \mathrm{m}]$ & $\mathrm{D}[\mu \mathrm{m}]$ & $\mathrm{d}[\mu \mathrm{m}]$ \\
\hline Structure 1 & 80 & 4.5 & 3.7 & 1.8 \\
\hline Structure 2 & 80 & 4.5 & 4.2 & 1.9 \\
\hline
\end{tabular}

Tab. 1. Geometrical parameters of designed structures.

COMSOL Multiphysics software based on the finite element method was used for numerical calculations of tension distribution generated by temperature and

*E-mail: wojcik@umcs.lublin.pl hydrostatic pressure. In the following step modified refractive index distribution was calculated and wave equations were solved.

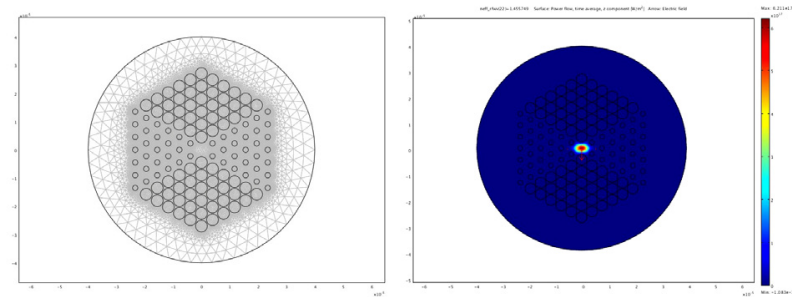

Fig. 1. Fiber structure and distribution of fundamental mode.

Figure 1 shows the designed fiber structure and calculated fundamental mode field distribution.

The software used makes it possible to calculate phase modal birefringence $B$, temperature sensitivity $K_{\mathrm{T}}$ and pressure sensitivity $K_{\mathrm{P}}$, which are defined in the following way:

$$
B=\frac{\lambda}{2 \pi}\left(\beta_{X}-\beta_{Y}\right)
$$

where $\beta \mathrm{x}$ and $\beta \mathrm{y}$ are propagation constants of the orthogonally polarized modes.

$$
K_{U}=\frac{1}{L} \frac{d\left(\phi_{X}-\phi_{Y}\right)}{d U}
$$

Phase sensitivity $\mathrm{K}_{\mathrm{U}}$ is defined as phase shift between polarizing modes, caused by parameter $U$ on optical fiber about length $L$. Parameters $\phi_{x}$ and $\phi_{y}$ are defined as the phase of polarizing mode OX and OY

The results of sensitivity calculations for temperature $K_{\mathrm{T}}$, pressure $K_{\mathrm{P}}$, birefrigence $B$, and calculated selectivity of pressure sensitivity to temperature sensitivity $\mathrm{S}_{\mathrm{P} / \mathrm{T}}$ are shown in Tab. 2

\begin{tabular}{|c|c|c|c|c|}
\hline Fiber no. & \multicolumn{2}{|c|}{ Fibre 1 } & \multicolumn{2}{c|}{ Fibre 2 } \\
\hline$\lambda[\mathrm{nm}]$ & 633 & 1550 & 633 & 1500 \\
\hline $\mathrm{K}_{\mathrm{P}}[\mathrm{rad} / \mathrm{MPa} \cdot \mathrm{m}]$ & 30 & 16 & 60 & 16 \\
\hline $\mathrm{K}_{\mathrm{T}}[\mathrm{rad} / \mathrm{K} \cdot \mathrm{m}]$ & $<0.04$ & $<0.04$ & $<0.04$ & $<0.04$ \\
\hline $\mathrm{B}$ & $4.8 \cdot 10^{-5}$ & $3.32 \cdot 10^{-4}$ & $7.5 \cdot 10^{-5}$ & $5.04 \cdot 10^{-4}$ \\
\hline $\mathrm{S}_{\mathrm{P} / \mathrm{T}}[\mathrm{K} / \mathrm{MPa}]$ & 750 & 400 & 1500 & 400 \\
\hline
\end{tabular}

Tab.2. Calculated values of $K_{P}, K_{T}$ and $B$. 
Calculations were done for a 633 and 1550nm wavelength. The temperature sensitivity value is $0 \mathrm{rad} / \mathrm{K} \cdot \mathrm{m}$ with a calculation accuracy of $0.04 \mathrm{rad} / \mathrm{K}^{*} \mathrm{~m}$. In order to calculate selectivity $S_{\mathrm{P} / \mathrm{T}}$ we utilize temperature sensitivity of $0.04 \mathrm{rad} / \mathrm{K}^{*} \mathrm{~m}$ as a lower limit of calculation accuracy.

In the Department of Optical Fibers Technology UMCS, V-type high birefrigent photonic optical fibres manufacturing technology was developed. Two fibres with structural parameters close to those chosen in the modeling process were produced. They were labeled $080421 \mathrm{P} 4$ (structure 1) and 080515P2 (structure 2), and are presented in the Fig. 2.
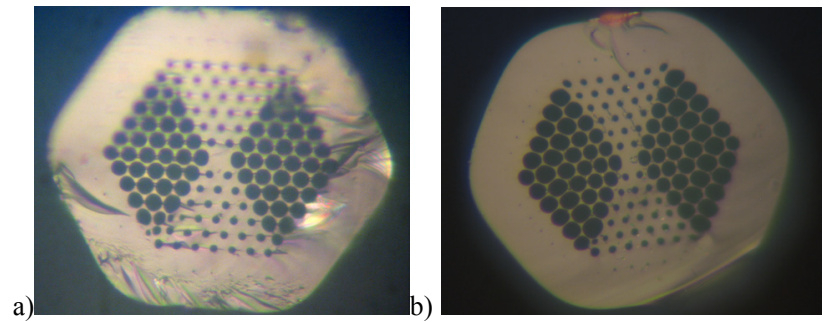

Fig. 2. Optical microscope photo of manufactured V-shape high birefringence photonic crystal fibers a) fiber no. 080421P4, b) fiber no. $080515 \mathrm{P} 2$.

For fabricated fibers sensitivity $K_{\mathrm{T}}$ and $K_{\mathrm{P}}[5,6]$ were measured in the interferometric setup. The schematic diagram of the setup is presented in Fig. 3. A He-Ne laser and a pigtailed $1550 \mathrm{~nm}$ semiconductor laser were used as the light sources.

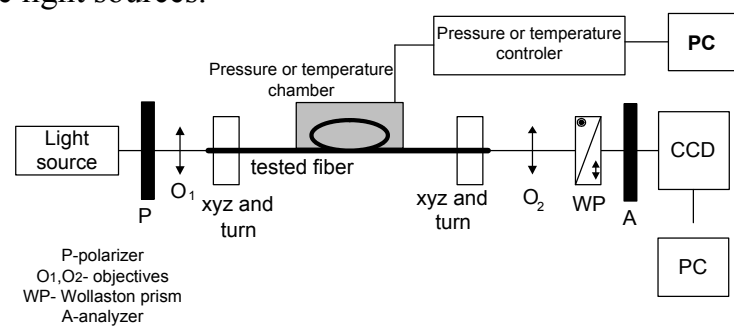

Fig. 3. Schematic diagram of the system for measuring temperature and pressure sensitivities.

For $K_{\mathrm{P}}$ sensitivity the pressure was changed in the range of $0,1 \div 6 \mathrm{MPa}$. The length of the fiber $\left(L_{\mathrm{P}}\right)$ in the pressure chamber was $1 \mathrm{~m} . K_{\mathrm{P}}$ value was calculated from the following formula:

$$
K_{P}=\frac{2 \pi}{L_{P}} \frac{\Delta M}{\Delta P}
$$

$\Delta M$ is the number of interference pattern fringes that shifted when the pressure was changed by $\Delta P$.
For $K_{\mathrm{T}}$ sensitivity measurements the temperature of fibre was changed in the range of $5 \div 95^{\circ} \mathrm{C}$. The length of the fibre $\left(L_{\mathrm{P}}\right)$ in the temperature chamber was about $5 \mathrm{~m}$. To avoid the coating's influence on $K_{\mathrm{T}}$ value, measurement was made for the fibers without a polymer coating [7]. Sensitivity value was calculated from the following equation:

$$
K_{T}=\frac{2 \pi}{L_{T}} \frac{\Delta M}{\Delta T}
$$

$\Delta M$ is the number of interference pattern fringes that shifted when the temperature was changed by $\Delta T$.

Table 2 shows obtained $K_{\mathrm{P}}$ and $K_{\mathrm{T}}$ values. Expected values obtained by extrapolation, based on the measurements of photonic crystal fiber with an asymmetric filling factor caused by two bigger holes placed on both sides of the core were marked with asterisk *. [1].

\begin{tabular}{|c|c|c|c|}
\hline Fiber no. & \multicolumn{2}{|c|}{$080421 \mathrm{P} 4$} & $080515 \mathrm{P} 2$ \\
\hline$\lambda[\mathrm{nm}]$ & 633 & 1550 & 633 \\
\hline $\mathrm{K}_{\mathrm{P}}[\mathrm{rad} / \mathrm{MPa} \cdot \mathrm{m}]$ & 44 & 16 & 80 \\
\hline $\mathrm{K}_{\mathrm{T}}[\mathrm{rad} / \mathrm{K} \cdot \mathrm{m}]$ & $10^{-2} *$ & $1,13 \cdot 10^{-2}$ & $10^{-2 *}$ \\
\hline $\mathrm{S}_{\mathrm{P} / \mathrm{T}}[\mathrm{K} / \mathrm{MPa}]$ & $4400^{*}$ & 1416 & $8000^{*}$ \\
\hline
\end{tabular}

Tab. 3. Measured temperature and pressure sensitivities

There is not much conformity between modelling and experimentally obtained results of pressure and temperature sensitivities. Table 4 presents measured geometrical parameters of the fabricated fibers. Those measurements were made with an optical microscope. The arithmetic means of the measured diameters of the holes are used in calculations.

\begin{tabular}{|c|c|c|c|}
\hline $\begin{array}{l}\text { No. of } \\
\text { optical } \\
\text { fiber }\end{array}$ & $\Lambda$ & $\begin{array}{c}\mathrm{d} \\
\text { diameters of } \\
\text { small holes } \\
\text { minimum and } \\
\text { maximum } \\
\text { measured } \\
\text { value }\end{array}$ & $\begin{array}{c}\mathrm{D} \\
\text { diameters of } \\
\text { large holes } \\
\text { minimum and } \\
\text { maximum } \\
\text { measured } \\
\text { value }\end{array}$ \\
\hline $\begin{array}{c}080515 \\
\mathrm{P} 2\end{array}$ & $4.7 \mu \mathrm{m}$ & $1.4 \mu \mathrm{m}-2.5 \mu \mathrm{m}$ & $4 \mu \mathrm{m}-5 \mu \mathrm{m}$ \\
\hline $\begin{array}{c}080421 \\
\mathrm{P} 4\end{array}$ & $4.6 \mu \mathrm{m}$ & $1.9 \mu \mathrm{m}-2.5 \mu \mathrm{m}$ & $3.8 \mu \mathrm{m}-5.7 \mu \mathrm{m}$ \\
\hline
\end{tabular}

Tab. 4. Measured geometrical parameters of the two fabricated V-shape photonic crystal fibers

However, the accuracy of optical measurements of holes diameters is not very high. Additionally, modelling we assumed that all small holes had the same diameters as well as large holes. In Figure 4 one can see that the above assumption is not correct. 


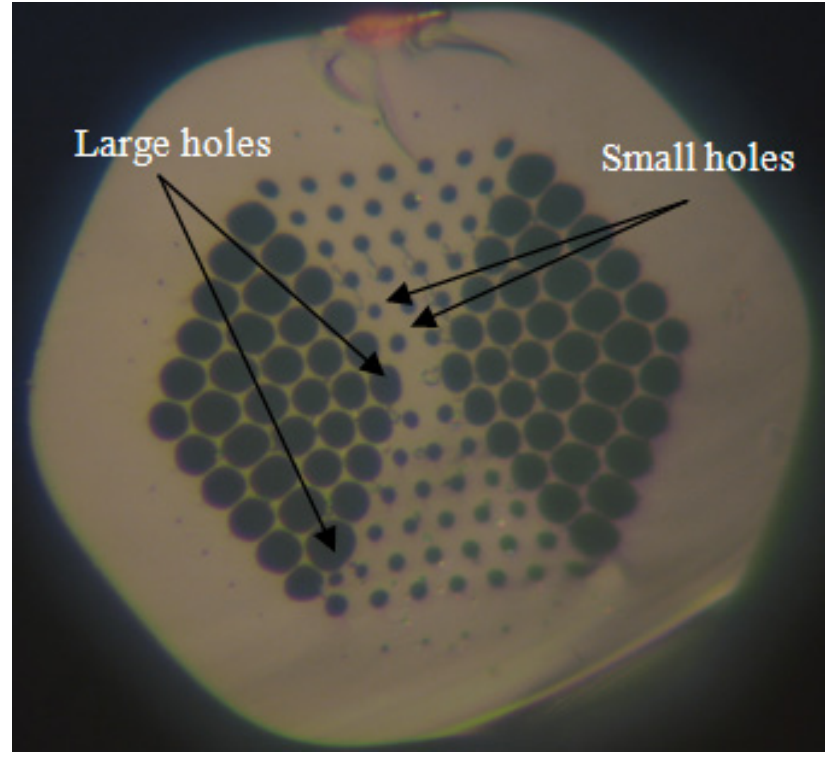

Fig. 4. PCF's fibre with different smaller holes diameter and larger holes diameter

The paper presents a new type of high birefringent photonic crystal optical fiber with enhanced sensitivity to pressure.

Technology of those V-type high birefringent photonic optical fibers was elaborated.

The calculations of pressure sensitivity, temperature sensitivity and birefringence were done with a finite element method.

Two fibers with structural parameters close to those chosen in the modeling process were produced.

Pressure and temperature sensitivities were measured.
Fabricated V-shape optical fibers have the selectivity one order of magnitude larger than the best side hole optical fiber [2].

These fibers has lower sensitivity to pressure therefore there will be a lower negative influence of protective coatings on temperature sensitivity in real application as we theoretically and experimentally have proved in [8].

Non-uniformities of diameters of both small and large holes probably don't have any significantly negative influence on sensing parameters of fabricated fibers as we experimentally confirmed in [4]. However this thesis must be confirmed in a modeling process.

The development of technology of those fibres allows uniform diameters of small and large holes.

Probably the results of modeling will conform better with the results of modeling measurements made for a real structure.

This work was financed with PHOSFOS Program and statutory investigation of Maria Curie Sklodowska University

\section{References}

[1] W. Bock, W Urbanczyk, J. Wojcik, , Proc. SPIE vol. 2341, 152159 (1994).

[2] J. Wojcik, et al., Proc. SPIE 3731, 88-93 (1999).

[3] M. Makara, et al. ,TAL 2008 Conference Technology and application of ligthquides, Poland (2008)

[4] J. Wojcik, et al., Network of Excellence on Micro Optics NEMO General Scientific Meeting, Karlsruhe 9-10, 02 (2006).

[5] G. Statkiewicz, T. Martynkien, W. Urbańczyk, Optics Communications 241, 339-348 (2004).

[6] T. Martynkien, et al., Opt Quant Electron 39, 481-489 (2007).

[7] J. Wójcik, et al., TAL96 Conference Technology and application of ligthquides, Poland (1996). 